\title{
Periodic BVPs for fractional order impulsive evolution equations
}

\author{
Xiulan $\mathrm{Yu}^{1}$ and JinRong Wang ${ }^{2,3^{*}}$
}

${ }^{\text {*Correspondence: }}$
wangjinrong@gznc.edu.cn
${ }^{2}$ Department of Mathematics,
Guizhou University, Guiyang,
Guizhou 550025, P.R. China
${ }^{3}$ School of Mathematics and
Computer Science, Guizhou Normal
College, Guiyang, Guizhou 550018,
P.R. China
Full list of author information is
available at the end of the article

"Correspondence:

wangjinrong@gznc.edu.cn

${ }^{3}$ School of Mathematics and

Computer Science, Guizhou Normal

P.R. China

available at the end of the article

\begin{abstract}
In this paper, we study periodic BVPs for fractional order impulsive evolution equations. The existence and boundedness of piecewise continuous mild solutions and design parameter drift for periodic motion of linear problems are presented. Furthermore, existence results of piecewise continuous mild solutions for semilinear impulsive periodic problems are showed. Finally, an example is given to illustrate the results.
\end{abstract}

MSC: 34B05; 34G10; 47D06

Keywords: fractional order; impulsive evolution equations; periodic BVPs

\section{Introduction}

In order to describe dynamics of populations subject to abrupt changes as well as other evolution processes such as harvesting, diseases, and so forth, many researchers have used impulsive differential systems to describe the model since the last century. For a wideranging bibliography and exposition on this important object see for instance the monographs of [1-4] and the papers [5-12].

Fractional differential equations appear naturally in fields such as viscoelasticity, electrical circuits, nonlinear oscillation of earthquakes etc. In particular, impulsive fractional evolution equations are used to describe many practical dynamical systems in many evolutionary processes models. Recently, Wang et al. [13] discussed Cauchy problems and nonlocal problems for impulsive fractional evolution equations involving the Caputo fractional derivative. However, periodic boundary value problems (BVPs for short) for impulsive fractional evolution equations have not been studied extensively.

In this paper we study the periodic BVPs for impulsive fractional evolution equations. Firstly, we discuss periodic BVPs for impulsive fractional evolution equations:

$$
\left\{\begin{array}{l}
{ }^{c} D_{0, t}^{q} x(t)=A x(t)+f(t), \quad q \in(0,1), t \in J=[0, T], t \neq t_{k}, \\
x(0)=x(T), \\
x\left(t_{k}^{+}\right)=x\left(t_{k}^{-}\right)+y_{k}, \quad k=1,2, \ldots, \delta,
\end{array}\right.
$$

in Banach space $X$, where ${ }^{c} D_{0, t}^{q}$ is the Caputo fractional derivative of order $q$ with the lower limit zero, $A: D(A) \subseteq X \rightarrow X$ is the generator of a $C_{0}$-semigroup $\{S(t), t \geq 0\}$ on a Banach space $X, f: J \rightarrow X$ is continuous, $x(0)$ and $y_{k}$ are the elements of $X, 0=t_{0}<t_{1}<t_{2}<\cdots<$ $t_{\delta}<t_{\delta+1}=T$, and $x\left(t_{k}^{+}\right)=\lim _{h \rightarrow 0^{+}}=x\left(t_{k}+h\right)$ and $x\left(t_{k}^{-}\right)=x\left(t_{k}\right)$ represent respectively the right and left limits of $x(t)$ at $t=t_{k}$.

C2014 Yu and Wang; licensee Springer. This is an Open Access article distributed under the terms of the Creative Commons Attribution License (http://creativecommons.org/licenses/by/2.0), which permits unrestricted use, distribution, and reproduction in any medium, provided the original work is properly cited. 
Secondly, we design parameter drift for the above periodic motion. We study the following impulsive periodic BVPs with parameter perturbations:

$$
\left\{\begin{array}{l}
{ }^{c} D_{0, t}^{q} x(t)=A x(t)+f(t)+p(t, x(t), \xi), \quad t \in J, t \neq t_{k}, \\
x(0)=x(T), \\
x\left(t_{k}^{+}\right)=x\left(t_{k}^{-}\right)+y_{k}, \quad k=1,2, \ldots, \delta
\end{array}\right.
$$

where $p$ is a given function and $\xi \in \Lambda=(-\tilde{\xi}, \tilde{\xi})$ is a small parameter perturbation that may be caused by some adaptive control algorithms or parameter drift.

Finally, we consider semilinear impulsive periodic problems:

$$
\left\{\begin{array}{l}
{ }^{c} D_{0, t}^{q} x(t)=A x(t)+f(t, x(t)), \quad t \in J, t \neq t_{k}, \\
x(0)=x(T), \\
x\left(t_{k}^{+}\right)=x\left(t_{k}^{-}\right)+I_{k}\left(x\left(t_{k}^{-}\right)\right), \quad k=1,2, \ldots, \delta,
\end{array}\right.
$$

where $f: J \times X \rightarrow X$ is continuous and $I_{k}: X \rightarrow X$ is continuous.

The rest of this paper is organized as follows. In Section 2, the existence and boundedness of the operator $B=[I-\mathcal{T}(T)]^{-1}$ are given. In Section 3, the existence and boundedness of $P C$-mild solutions and the design parameter drift for such a periodic motion are presented. In Section 4, existence results of $P C$-mild solutions for impulsive periodic problems are showed. Finally, an example is presented to illustrate the theory.

\section{Existence and boundedness of operator $B=[I-\mathcal{T}(T)]^{-1}$}

Suppose $T>0$, let $J=[0, T]$. Let $M=\sup _{t \geq 0}\|S(t)\|<\infty$. We denote by $C(J, X)$ the Banach space of all continuous functions from $J$ into $X$ with the norm $\|x\|_{C}=\sup \{\|x(t)\|: t \in J\}$. We also introduce the set of functions $P C(J, X)=\{x: J \rightarrow X: x$ is continuous at $t \in$ $J \backslash\left\{t_{1}, t_{2}, \ldots, t_{\delta}\right\}$, and $x$ is continuous from the left and has right-hand limits at $t \in$ $\left.\left\{t_{1}, t_{2}, \ldots, t_{\delta}\right\}\right\}$. Endowed with the norm

$$
\|x\|_{P C}=\max \left\{\sup _{t \in J}\|x(t+0)\|, \sup _{t \in J}\|x(t-0)\|\right\},
$$

it is easy to see $\left(P C(J, X),\|\cdot\|_{P C}\right)$ is a Banach space.

For measurable functions $l: J \rightarrow R$, define the norm $\|l\|_{L^{p}(, R)}=\left(\int_{J}|l(t)|^{p} d t\right)^{\frac{1}{p}}, 1 \leq p<\infty$. We denote by $L^{p}(J, R)$ the Banach space of all Lebesgue measurable functions $l$ with $\|l\|_{L^{p}(, R)}<\infty$.

Definition 2.1 ([14]) The fractional integral of order $\gamma$ with the lower limit zero for a function $f$ is defined as

$$
I_{0, t}^{\gamma} f(t)=\frac{1}{\Gamma(\gamma)} \int_{0}^{t} \frac{f(s)}{(t-s)^{1-\gamma}} d s, \quad t>0, \gamma>0,
$$

provided the right side is point-wise defined on $[0, \infty)$, where $\Gamma(\cdot)$ is the gamma function.

Definition 2.2 ([14]) The Riemann-Liouville derivative of order $\gamma$ with the lower limit zero for a function $f:[0, \infty) \rightarrow R$ can be written as

$$
{ }^{L} D_{0, t}^{\gamma} f(t)=\frac{1}{\Gamma(n-\gamma)} \frac{d^{n}}{d t^{n}} \int_{0}^{t} \frac{f(s)}{(t-s)^{\gamma+1-n}} d s, \quad t>0, n-1<\gamma<n .
$$


Definition 2.3 ([14]) The Caputo derivative of order $\gamma$ for a function $f:[0, \infty) \rightarrow R$ can be written as

$$
{ }^{c} D_{0, t}^{\gamma} f(t)={ }^{L} D_{0, t}^{\gamma}\left(f(t)-\sum_{k=0}^{n-1} \frac{t^{k}}{k !} f^{(k)}(0)\right), \quad t>0, n-1<\gamma<n .
$$

Remark 2.4 If $f$ is an abstract function with values in $X$, then the integrals which appear in Definitions 2.1 and 2.2 are taken in Bochner's sense.

As in our previous work [13], by a $P C$-mild solution of (1) we mean the function $x \in$ $P C(J, X)$ satisfying

$$
\begin{aligned}
x(t)= & \mathcal{T}(t) x(0)+\sum_{i=1}^{k} \mathcal{T}\left(t-t_{i}\right) y_{i} \\
& +\int_{0}^{t}(t-s)^{q-1} s(t-s) f(s) d s, \quad t \in\left(t_{i}, t_{i+1}\right], k=1,2, \ldots, \delta,
\end{aligned}
$$

and $x(0)=x(T)$, where

$$
\mathcal{T}(t)=\int_{0}^{\infty} \xi_{q}(\theta) S\left(t^{q} \theta\right) d \theta, \quad s(t)=q \int_{0}^{\infty} \theta \xi_{q}(\theta) S\left(t^{q} \theta\right) d \theta
$$

and

$$
\begin{aligned}
& \xi_{q}(\theta)=\frac{1}{q} \theta^{-1-\frac{1}{q}} \vartheta_{q}\left(\theta^{-\frac{1}{q}}\right) \geq 0, \\
& \vartheta_{q}(\theta)=\frac{1}{\pi} \sum_{n=1}^{\infty}(-1)^{n-1} \theta^{-q n-1} \frac{\Gamma(n q+1)}{n !} \sin (n \pi q), \quad \theta \in(0, \infty),
\end{aligned}
$$

here $\xi_{q}$ is a probability density function defined on $(0, \infty)$, that is,

$$
\xi_{q}(\theta) \geq 0, \quad \theta \in(0, \infty) \quad \text { and } \quad \int_{0}^{\infty} \xi_{q}(\theta) d \theta=1 .
$$

Lemma 2.5 (see Lemma 2.9 [15]) The operator $\mathcal{T}$ has the following properties:

(i) For any fixed $t \geq 0, \mathcal{T}(t)$ and $\&(t)$ are linear and bounded operators, i.e., for any $x \in X,\|\mathcal{T}(t) x\| \leq M\|x\|$ and $\|\delta(t) x\| \leq \frac{M}{\Gamma(q)}\|x\|$.

(ii) Both $\{\delta(t), t \geq 0\}$ and $\{\mathcal{T}(t), t \geq 0\}$ are strongly continuous.

(iii) For every $t>0, \mathcal{T}(t)$ and $\delta(t)$ are compact operators if $S(t)$ is compact.

Suppose that here the bounded operator $B: X \rightarrow X$ exists given by

$$
B=[I-\mathcal{T}(T)]^{-1} .
$$

We present sufficient conditions for the existence and boundedness of the operator $B$.

Lemma 2.6 (see Theorem 3.3 and Remark 3.4 [16]) The operator B defined in (4) exists and is bounded, if one of the following three conditions holds: 
(i) $S(t)$ is compact for each $t>0$ and the homogeneous linear nonlocal problem

$$
\left\{\begin{array}{l}
{ }^{c} D_{0, t}^{q} x(t)=A x(t), \quad t \in J \\
x(0)=x(T)
\end{array}\right.
$$

has no non-trivial PC-mild solutions.

(ii) If $\|\mathcal{T}(T)\|<1$, then the operator $I-\mathcal{T}(T)$ is invertible and $[I-\mathcal{T}(T)]^{-1} \in L_{b}(X)$.

(iii) If $\|S(t)\|<1$ for $t \in(0, T]$, then $\mathcal{T}(n T) \rightarrow 0$ as $n \rightarrow \infty$ and the operator $I-\mathcal{T}(T)$ is invertible and $[I-\mathcal{T}(T)]^{-1} \in L_{b}(X)$.

\section{Linear impulsive periodic problems and robustness}

In this section, we consider the existence of $P C$-mild solutions of (1) and of design parameter drift for (2).

We list the following assumptions.

(HA): $A: D(A) \rightarrow X$ is the infinitesimal generator of a $C_{0}$-semigroup $\{S(t), t \geq 0\}$.

(HF): $f: J \rightarrow X$ is strongly measurable for $t \in J$ and there exist a constant $q_{1} \in(0, q)$ and a real-valued function $h(\cdot) \in L^{\frac{1}{q_{1}}}(J, R)$ such that $\|f(t)\| \leq h(t)$, for each $t \in J$.

(HB): The operator $B$ defined in (4) exists and is bounded.

We first give an existence theorem of $P C$-mild solutions of (1).

Theorem 3.1 Assume that (HA), (HF), and (HB) are satisfied. Then (1) has a PC-mild solution given by

$$
\begin{aligned}
x_{T}(t)= & \mathcal{T}(t) B z+\sum_{i=1}^{k} \mathcal{T}\left(t-t_{i}\right) y_{i} \\
& +\int_{0}^{t}(t-s)^{q-1} \delta(t-s) f(s) d s, \quad t \in\left(t_{i}, t_{i+1}\right], k=1,2, \ldots, \delta,
\end{aligned}
$$

where

$$
z=\sum_{i=1}^{\delta} \mathcal{T}\left(t-t_{i}\right) y_{i}+\int_{0}^{T}(T-s)^{q-1} \delta(T-s) f(s) d s .
$$

Further, we have

$$
\left\|x_{T}(t)\right\| \leq M\left(M L_{1}+1\right)\left[\sum_{i=1}^{\delta}\left\|y_{i}\right\|+\frac{T^{q-q_{1}} H}{\Gamma(q)\left(\frac{q-q_{1}}{1-q_{1}}\right)^{1-q_{1}}}\right]
$$

where $L_{1}=\|B\|$ and $H=\|h\|_{L^{\frac{1}{q_{1}}}(J, R)}$.

Proof We consider an impulsive Cauchy problem,

$$
\left\{\begin{array}{l}
{ }^{c} D_{0, t}^{q} x(t)=A x(t)+f(t), \quad t \in J, t \neq t_{k}, \\
x(0)=B z:=\bar{x}, \quad z \text { defined in }(6), \\
x\left(t_{k}^{+}\right)=x\left(t_{k}^{-}\right)+y_{k}, \quad k=1,2, \ldots, \delta .
\end{array}\right.
$$

It follows from the expression of the initial value $x(0)=B z:=\bar{x}$ that the mild solution $x_{T}(\cdot)$ of (8) corresponding to the initial value $\bar{x}$ must be the $P C$-mild solution of (1). 
For the estimation of $x_{T}(\cdot)$, by Lemma 2.5 ,

$$
\begin{aligned}
\left\|x_{T}(t)\right\| \leq & \|\mathcal{T}(t) B z\|+\sum_{i=1}^{k}\left\|\mathcal{T}\left(t-t_{i}\right)\right\|\left\|y_{i}\right\|+\int_{0}^{t}(t-s)^{q-1}\|s(t-s) f(s)\| d s \\
\leq & M\|B\|\|z\|+M \sum_{i=1}^{k}\left\|y_{i}\right\|+\frac{M}{\Gamma(q)} \int_{0}^{t}(t-s)^{q-1} h(s) d s \\
\leq & M\|B\|\left(\sum_{i=1}^{\delta}\left\|\mathcal{T}\left(t-t_{i}\right)\right\|\left\|y_{i}\right\|+\int_{0}^{T}(T-s)^{q-1}\|s(T-s) f(s)\| d s\right) \\
& +M \sum_{i=1}^{\delta}\left\|y_{i}\right\|+\frac{M}{\Gamma(q)} \int_{0}^{t}(t-s)^{q-1} h(s) d s \\
\leq & M^{2}\|B\|\left(\sum_{i=1}^{\delta}\left\|y_{i}\right\|+\frac{1}{\Gamma(q)} \int_{0}^{T}(T-s)^{q-1} h(s) d s\right) \\
& +M \sum_{i=1}^{\delta}\left\|y_{i}\right\|+\frac{M}{\Gamma(q)} \int_{0}^{t}(t-s)^{q-1} h(s) d s \\
\leq & M^{2}\|B\|\left[\sum_{i=1}^{\delta}\left\|y_{i}\right\|+\frac{1}{\Gamma(q)}\left(\int_{0}^{T}(T-s)^{\frac{q-1}{1-q_{1}}} d s\right)^{1-q_{1}}\left(\int_{0}^{T} h^{\frac{1}{q_{1}}}(s) d s\right)^{q_{1}}\right] \\
& +\left[M \sum_{i=1}^{\delta}\left\|y_{i}\right\|+\frac{M}{\Gamma(q)}\left(\int_{0}^{t}(t-s)^{\frac{q-1}{1-q_{1}}} d s\right)^{1-q_{1}}\left(\int_{0}^{t} h^{\frac{1}{q_{1}}}(s) d s\right)^{q_{1}}\right] \\
\leq & M\left(M L_{1}+1\right)\left[\sum_{i=1}^{\delta}\left\|y_{i}\right\|+\frac{T^{q-q_{1}} H}{\Gamma(q)\left(\frac{q-q_{1}}{1-q_{1}}\right)^{1-q_{1}}}\right],
\end{aligned}
$$

where $L_{1}=\|B\|$ and $H=\|h\|_{L^{\frac{1}{q_{1}}}(J, R)}$. The desired results are obtained.

Remark 3.2 In Theorem 3.1, we replace (HB) by $\{S(t), t \geq 0\}$; it is a compact semigroup and

$$
\left\{\begin{array}{l}
{ }^{c} D_{0, t}^{q} x(t)=A x(t), \quad t \in J, \\
x(0)=x(T),
\end{array}\right.
$$

has no non-trivial mild solutions. Then one can use the Fredholm alternative theorem to derive that the operator equation $[I-\mathcal{T}(T)] x(0)=z$ has a unique solution $x(0)=[I-$ $\mathcal{T}(T)]^{-1} z:=\bar{x}$. Thus, the $P C$-mild solution of (1) is unique.

Define $P C_{T}(J, X)=\{x \in P C(J, X): x(0)=x(T)\}$ with $\|x\|_{P C_{T}}=\sup \{\|x(t)\|: t \in J\}$ for $x \in$ $P C_{T}(J, X)$. It can be seen that endowed with the norm $\|\cdot\|_{P C_{T}}, P C_{T}(J, X)$ is a Banach space. Denote

$$
\begin{aligned}
& \mathcal{S}_{\rho}=\left\{x \in P C_{T}(J, X):\|x\|_{P C_{T}} \leq \rho\right\}, \\
& \mathcal{B}\left(x, \rho_{1}\right)=\left\{x \in P C_{T}(J ; X):\left\|x-x_{T}\right\|_{P C_{T}} \leq \rho_{1}\right\},
\end{aligned}
$$


where

$$
\begin{aligned}
& \rho=M\left(M L_{1}+1\right)\left[\sum_{i=1}^{\delta}\left\|y_{i}\right\|+\frac{T^{q-q_{1}} H}{\Gamma(q)\left(\frac{q-q_{1}}{1-q_{1}}\right)^{1-q_{1}}}+\frac{T^{q}}{\Gamma(q+1)} \sup _{|\xi| \leq \tilde{\xi}} \chi(\xi)\right], \\
& \rho_{1}=\frac{M\left(M L_{1}+1\right) T^{q}}{\Gamma(1+q)} \sup _{|\xi| \leq \tilde{\xi}} \chi(\xi),
\end{aligned}
$$

and $\chi$ is a nonnegative function.

We introduce the assumption (HP):

(HP1): $p: J \times \mathcal{S}_{\rho} \times \Lambda \rightarrow X$ is measurable in $t$.

(HP2): There exists a nonnegative function $\varpi$ such that $\lim _{\xi \rightarrow 0} \varpi(\xi)=\varpi(0)=0$ and for any $t \in J, x, y \in \mathcal{S}_{\rho}$, and $\xi \in \Lambda$, and we have

$$
\|p(t, x, \xi)-p(t, y, \xi)\| \leq \varpi(\xi)\|x-y\| .
$$

(HP3): There exists a nonnegative function $\chi$ such that $\lim _{\xi \rightarrow 0} \chi(\xi)=\chi(0)=0$, and for any $t \in J, x \in \mathcal{S}_{\rho}$, and $\xi \in \Lambda$, we have

$$
\|p(t, x, \xi)\| \leq \chi(\xi)
$$

By a $P C$-mild solution of (2), we mean the function $x \in P C(J, X)$ satisfying

$$
\begin{aligned}
x(t)= & \mathcal{T}(t) x(0)+\sum_{i=1}^{k} \mathcal{T}\left(t-t_{i}\right) y_{i} \\
& +\int_{0}^{t}(t-s)^{q-1} s(t-s)[f(s)+p(s, x(s), \xi)] d s, \quad k=1,2, \ldots, \delta,
\end{aligned}
$$

and $x(0)=x(T)$.

The following result shows that given a periodic motion we can design periodic motion controllers that are robust with respect to parameter drift.

Theorem 3.3 Let (HA), (HF), (HB), and (HP) hold. Then there is a $\xi_{0} \in(0, \tilde{\xi})$ such that, for $|\xi| \leq \xi_{0}$, (2) has a PC-mild solution $x_{T}^{\xi}$ satisfying

$$
\left\|x_{T}^{\xi}-x_{T}\right\|_{P C_{T}} \leq \rho_{1}
$$

and $\lim _{\xi \rightarrow 0} x_{T}^{\xi}(t)=x_{T}(t)$ uniformly on $t \in J$ where $x_{T}$ is the mild solution of (1).

Proof By (HB), one can choose

$$
x_{0}=B\left[z+\int_{0}^{T}(T-s)^{q-1} s(T-s) p(s, x(s), \xi) d s\right] \in X
$$

to be fixed. Consider the map $\mathcal{O}$ on $\mathcal{B}\left(x_{T}, \rho_{1}\right)$ given by

$$
(\mathcal{O} x)(t)=\mathcal{T}(t) x_{0}+\sum_{i=1}^{k} \mathcal{T}\left(t-t_{i}\right) y_{i}+\int_{0}^{t}(t-s)^{q-1} s(t-s)[f(s)+p(s, x(s), \xi)] d s .
$$

Obviously, $\mathcal{O} x \in P C_{T}(J, X)$. 
By the assumption (HP), we can choose a $\xi_{0} \in(0, \tilde{\xi})$ such that

$$
\frac{M\left(M L_{1}+1\right) T^{q}}{\Gamma(1+q)} \sup _{|\xi| \leq \xi_{0}} \chi(\xi) \leq \rho_{1}
$$

and

$$
\eta=\frac{M\left(M L_{1}+1\right) T^{q}}{\Gamma(1+q)} \sup _{|\xi| \leq \xi_{0}} \varpi(\xi)<1 .
$$

For $\xi \in\left(-\xi_{0}, \xi_{0}\right)$ and $x, y \in \mathcal{B}\left(x_{T}, \rho_{1}\right)$, one can verify that

$$
\left\|\mathcal{O} x-x_{T}\right\|_{P C_{T}} \leq \frac{M\left(M L_{1}+1\right) T^{q}}{\Gamma(1+q)} \sup _{|\xi| \leq \xi_{0}} \chi(\xi) \leq \rho_{1}
$$

and

$$
\|\mathcal{O} x-\mathcal{O} y\|_{P C_{T}} \leq \eta\|x-y\|_{P C_{T}} .
$$

This implies that $\mathcal{O}$ is a contraction mapping on $\mathcal{B}\left(x_{T}, \rho_{1}\right)$. Then, $\mathcal{O}$ has a unique fixed point $x_{T}^{\xi} \in \mathcal{B}\left(x_{T}, \rho_{1}\right)$ given by

$$
x_{T}^{\xi}(t)=\mathcal{T}(t) x_{0}+\int_{0}^{t}(t-s)^{q-1} \delta(t-s)\left[f(s)+p\left(s, x_{T}^{\xi}(s), \xi\right)\right] d s
$$

which is just the $P C$-mild solution of (2).

From the expressions (9) and (10), one can get $\left\|x_{T}^{\xi}-x_{T}\right\|_{P C_{T}} \leq \rho_{1}$. It is easy to see that $\lim _{\xi \rightarrow 0} x_{T}^{\xi}(t)=x_{T}(t)$ uniformly on $t \in J$.

\section{Semilinear impulsive periodic problems}

We impose the following assumptions.

(HF1): $f: J \times X \rightarrow X$ is continuous and there exist a constant $q_{1} \in(0, q)$ and a real-valued function $L_{f}(\cdot) \in L^{\frac{1}{q_{1}}}\left(J, R^{+}\right)$such that $\|f(t, x)-f(t, y)\| \leq L_{f}(t)\|x-y\|$ for all $x, y \in X$.

(HF2): $f: J \times X \rightarrow X$ is continuous and maps a bounded set into a bounded set.

(HF3): For each $x_{0} \in X$, there exists a constant $r>0$ such that

$$
M\left(M L_{1}+1\right)\left(\delta M_{I}+\frac{T^{q}}{\Gamma(1+q)} \sup _{s \in J, \phi \in Y_{\Gamma}}\|f(s, \phi(s))\|\right) \leq r,
$$

where

$$
Y_{\Gamma}=\{\phi \in P C(J, X):\|\phi\| \leq r \text { for } t \in J\} .
$$

(HI1): $I_{k}: X \rightarrow X$ is continuous and there exists a constant $L_{I}>0$ such that $\left\|I_{k}(x)-I_{k}(y)\right\| \leq L_{I}\|x-y\|$ for all $x, y \in X, k=1,2, \ldots, \delta$.

(HI2): $I_{k}: X \rightarrow X$ is continuous and there exists a constant $M_{I}>0$ such that $\left\|I_{k}(x)\right\| \leq M_{I}$, for all $x \in X, k=1,2, \ldots, \delta$. 
Theorem 4.1 Let (HA), (HB), (HI1), and (HF1) be satisfied. Then (3) has a unique PC-mild solution on J provided that

$$
M\left(M L_{1}+1\right)\left(\delta L_{I}+\frac{T^{*}}{\Gamma(q)}\right)<1, \quad T^{*}=\left[\left(\frac{1-q_{1}}{q-q_{1}}\right) T^{\frac{q-q_{1}}{1-q_{1}}}\right]^{1-q_{1}}\left\|L_{f}\right\|_{L^{\frac{1}{q_{1}}}\left(J, R^{+}\right)} .
$$

Proof Consider

$$
\left\{\begin{array}{l}
{ }^{c} D_{0, t}^{q} x(t)=A x(t)+f(t, x(t)), \quad t \in J, t \neq t_{k}, \\
x(0)=B z_{1}, \\
x\left(t_{k}^{+}\right)=x\left(t_{k}^{-}\right)+I_{k}\left(x\left(t_{k}^{-}\right)\right), \quad k=1,2, \ldots, \delta,
\end{array}\right.
$$

where

$$
z_{1}=\sum_{i=1}^{\delta} \mathcal{T}\left(t-t_{i}\right) I_{i}\left(x\left(t_{i}^{-}\right)\right)+\int_{0}^{T}(T-s)^{q-1} s(T-s) f(s, x(s)) d s,
$$

and we define an operator $Q$ on $P C(J, X)$ :

$$
\begin{aligned}
(Q x)(t)= & \mathcal{T}(t) B z_{1}+\sum_{i=1}^{k} \mathcal{T}\left(t-t_{i}\right) I_{i}\left(x\left(t_{i}^{-}\right)\right) \\
& +\int_{0}^{t}(t-s)^{q-1} s(t-s) f(s, x(s)) d s, \quad t \in\left(t_{i}, t_{i+1}\right], k=1,2, \ldots, \delta .
\end{aligned}
$$

Clearly, $Q$ is well defined on $P C(J, X)$ due to our assumptions.

Then, we only need to show that $Q$ is a contraction on $P C(J, X)$.

In general, for each $t \in\left(t_{k}, t_{k+1}\right], k=1,2, \ldots, \delta$ we have

$$
\begin{aligned}
\|(Q x)(t)-(Q y)(t)\| \\
\leq M^{2} L_{1} L_{I} \sum_{i=1}^{\delta}\left\|x\left(t_{i}^{-}\right)-y\left(t_{i}^{-}\right)\right\|+M L_{I} \sum_{i=1}^{k}\left\|x\left(t_{i}^{-}\right)-y\left(t_{i}^{-}\right)\right\| \\
\quad+\frac{M}{\Gamma(q)}\left(M L_{1} \int_{0}^{T}(T-s)^{q-1} L_{f}(s)\|x(s)-y(s)\| d s\right. \\
\left.\quad+\int_{0}^{t}(t-s)^{q-1} L_{f}(s)\|x(s)-y(s)\| d s\right) \\
\leq M L_{I}\left(M L_{1}+1\right) \delta\|x-y\|_{P C} \\
\quad+\frac{M\|x-y\|_{P C}}{\Gamma(q)}\left(M L_{1} \int_{0}^{T}(T-s)^{q-1} L_{f}(s) d s+\int_{0}^{t}(t-s)^{q-1} L_{f}(s) d s\right) \\
\leq M L_{I}\left(M L_{1}+1\right) \delta\|x-y\|_{P C} \\
\quad+\frac{M\|x-y\|_{P C}}{\Gamma(q)}\left[M L_{1}\left(\int_{0}^{T}(T-s)^{\frac{q-1}{1-q_{1}}} d s\right)^{1-q_{1}}\left\|L_{f}\right\|_{L^{\frac{1}{q_{1}}}\left(J, R^{+}\right)}\right. \\
\left.\quad+\left(\int_{0}^{t}(t-s)^{\frac{q-1}{1-q_{1}}} d s\right)^{1-q_{1}}\left\|L_{f}\right\|_{L^{\frac{1}{q_{1}}}\left(\left[0, t_{k+1}\right], R^{+}\right)}\right] \\
\leq M\left(M L_{1}+1\right)\left(\delta L_{I}+\frac{T^{*}}{\Gamma(q)}\right)^{\|x-y\|_{P C} .}
\end{aligned}
$$


Hence, the condition (11) allows us to conclude, in view of the Banach contraction mapping principle, that $Q$ has a unique fixed point $x \in P C(J, X)$, which is just the unique $P C$-mild solution of (3).

Theorem 4.2 Suppose that (HA), (HB), (HI2), and (HF2) and (HF3) are satisfied. Then for every $x_{0} \in X$, (3) has at least a PC-mild solution on $J$.

Proof Consider the mapping

$$
Q: P C(J, X) \rightarrow P C(J, X)
$$

by

$$
(Q v)(t)=\left(Q_{1} v\right)(t)+\left(Q_{2} v\right)(t)
$$

where

$$
\left(Q_{1} v\right)(t)=\mathcal{T}(t) B z_{1}+\int_{0}^{t}(t-s)^{q-1} \delta(t-s) f(s, v(s)) d s, \quad t \in J \backslash\left\{t_{1}, t_{2}, \ldots, t_{\delta}\right\} ;
$$

$z_{1}$ is defined in (13) and

$$
\left(Q_{2} v\right)(t)= \begin{cases}0, & t \in\left[0, t_{1}\right], \\ \sum_{i=1}^{k} \mathcal{T}\left(t-t_{i}\right) I_{i}\left(v\left(t_{i}^{-}\right)\right), & t \in\left(t_{k}, t_{k+1}\right], k=1, \ldots, \delta .\end{cases}
$$

For each $t \in\left(t_{k}, t_{k+1}\right], v \in Y_{\Gamma}$,

$$
\begin{aligned}
\|(Q v)(t)\| & \leq\left\|\left(Q_{1} v\right)(t)\right\|+\left\|\left(Q_{2} v\right)(t)\right\| \\
& \leq M\left(M L_{1}+1\right)\left(\delta M_{I}+\frac{T^{q}}{\Gamma(1+q)} \sup _{s \in J, \phi \in Y_{\Gamma}}\|f(s, \phi(s))\|\right) .
\end{aligned}
$$

Thus, we see that $Q: Y_{\Gamma} \rightarrow Y_{\Gamma}$.

Just like the proof in our previous work [13], one can prove that $Q$ is a continuous mapping from $Y_{\Gamma}$ to $Y_{\Gamma}$ and it is a compact operator. Now, Schauder's fixed point theorem implies that $Q$ has a fixed point, which gives rise to a $P C$-mild solution.

\section{Example}

We consider impulsive fractional differential equations with periodic boundary conditions,

$$
\left\{\begin{array}{l}
{ }^{c} D_{0, t}^{\frac{1}{2}} x(t, y)=\frac{\partial^{2}}{\partial y^{2}} x(t, y)+\frac{|x(t, y)|}{\gamma\left(1+9 e^{t}\right)(1+|x(t, y)|)}, \quad y \in(0, \pi), t \in\left[0, \frac{1}{2}\right) \cup\left(\frac{1}{2}, 1\right], \\
x(t, 0)=x(t, \pi)=0, \\
x(0, y)=x(1, y), \quad y \in(0, \pi), \\
x\left(\frac{1}{2}^{+}\right)=x\left(\frac{1}{2}^{-}\right)+\frac{\left|x\left(\frac{1}{2}^{-}, y\right)\right|}{\gamma(9+t)\left(1+\left|x\left(\frac{1}{2}, y\right)\right|\right)}, \quad y \in(0, \pi),
\end{array}\right.
$$

in $X=L^{2}(0, \pi)$ where $\gamma>0$ will be chosen later. 
Define $A x=-\frac{\partial^{2}}{\partial y^{2}} x$ for $x \in D(A)$ where $D(A)=\left\{x \in X: \frac{\partial x}{\partial y}, \frac{\partial^{2} x}{\partial y^{2}} \in X\right.$ and $\left.x(0)=x(\pi)=0\right\}$. Then $A$ is the infinitesimal generator of a $C_{0}$-semigroup $\{T(t), t \geq 0\}$ in $L^{2}(0, \pi)$. Moreover, $T(\cdot)$ is also compact and $\|T(t)\| \leq e^{-t} \leq 1, t \geq 0$. By the Fredholm alternative theorem, $[I-\mathcal{T}(1)]^{-1}$ exists and is bounded where $\mathcal{T}(\cdot)$ is defined in Section 2.

Define $f(t, x(t))(y)=\frac{|x(t, y)|}{\gamma\left(1+9 e^{t}\right)(1+|x(t, y)|)}, t \in\left[0, \frac{1}{2}\right) \cup\left(\frac{1}{2}, 1\right], x \in X, y \in(0, \pi)$ and $f:[0,1] \times X \rightarrow$

$X$ is a continuous function, $\left\|f\left(t, x_{1}\right)-f\left(t, x_{2}\right)\right\| \leq L_{f}\left\|x_{1}-x_{2}\right\|$, with $L_{f}=\frac{1}{10 \gamma} \in L^{\frac{1}{4}}\left([0,1], R^{+}\right)$.

Define $I_{1}\left(x\left(\frac{1}{2}^{-}\right)\right)(y)=\frac{\left|x\left(\frac{1}{2}^{-}, y\right)\right|}{\gamma(9+t)\left(1+\left|x\left(\frac{1}{2}^{-}, y\right)\right|\right)}, x \in X, y \in(0, \pi) .\left\|I_{1}\left(x_{1}\right)-I_{1}\left(x_{2}\right)\right\| \leq L_{I}\left\|x_{1}-x_{2}\right\|$ for all $x_{1}, x_{2} \in X$ with $L_{I}=\frac{1}{10 \gamma}$.

Moreover,

$$
M\left(M L_{1}+1\right)\left(\delta L_{I}+\frac{T^{*}}{\Gamma(q)}\right)=\left\|[I-\mathcal{T}(1)]^{-1}\right\|\left(\frac{1}{10 \gamma}+\frac{\sqrt[4]{27}}{10 \gamma \Gamma\left(\frac{1}{2}\right)}\right)<\frac{2}{5 \gamma}\left\|[I-\mathcal{T}(1)]^{-1}\right\|
$$

Thus, one can choose $\gamma>\frac{2}{5}\left\|[I-\mathcal{T}(1)]^{-1}\right\|$ such that (11) holds. Therefore, (17) has a unique $P C$-mild solution on $[0,1]$.

Competing interests

The authors declare that they have no competing interests.

\section{Authors' contributions}

This work was carried out in collaboration between all authors. JRW raised these interesting problems in this research. JRW and XLY proved the theorems, interpreted the results and wrote the article. All authors defined the research theme, read and approved the manuscript.

\section{Author details}

${ }^{1}$ College of Applied Mathematics, Shanxi University of Finance and Economics, Taiyuan, Shanxi 030031, P.R. China. ${ }^{2}$ Department of Mathematics, Guizhou University, Guiyang, Guizhou 550025, P.R. China. ${ }^{3}$ School of Mathematics and Computer Science, Guizhou Normal College, Guiyang, Guizhou 550018, P.R. China.

\section{Acknowledgements}

This work is partially supported by Key Support Subject (Applied Mathematics), Key Project on the Reforms of Teaching Contents, Course System of Guizhou Normal College and Doctor Project of Guizhou Normal College (13BS010) and Guizhou Province Education Planning Project (2013A062).

Received: 26 November 2013 Accepted: 16 January 2014 Published: 07 Feb 2014

\section{References}

1. Bainov, DD, Simeonov, PS: Impulsive Differential Equations: Periodic Solutions and Applications. Longman, New York (1993)

2. Samoilenko, AM, Perestyuk, NA: Impulsive Differential Equations. World Scientific Series on Nonlinear Science. Series A: Monographs and Treatises, vol. 14. World Scientific, Singapore (1995)

3. Benchohra, M, Henderson, J, Ntouyas, SK: Impulsive Differential Equations and Inclusions, vol. 2. Hindawi Publishing Corporation, New York (2006)

4. Lakshmikantham, V, Bainov, DD, Simeonov, PS: Theory of Impulsive Differential Equations. World Scientific, Singapore (1989)

5. Abada, N, Benchohra, M, Hammouche, H: Existence and controllability results for nondensely defined impulsive semilinear functional differential inclusions. J. Differ. Equ. 246, 3834-3863 (2009)

6. Ahmed, NU: Existence of optimal controls for a general class of impulsive systems on Banach space. SIAM J. Control Optim. 42, 669-685 (2003)

7. Akhmet, MU: On the smoothness of solutions of impulsive autonomous systems. Nonlinear Anal. TMA 60, $311-324$ (2005)

8. Fan, Z, Li, G: Existence results for semilinear differential equations with nonlocal and impulsive conditions. J. Funct. Anal. 258, 1709-1727 (2010)

9. Liang, J, Liu, JH, Xiao, T-J: Nonlocal impulsive problems for nonlinear differential equations in Banach spaces. Math. Comput. Model. 49, 798-804 (2009)

10. Liu, J: Nonlinear impulsive evolution equations. Dyn. Contin. Discrete Impuls. Syst. 6, 77-85 (1999)

11. Battelli, F, Fečkan, M: Chaos in singular impulsive O.D.E. Nonlinear Anal. TMA 28, 655-671 (1997)

12. Wang, J, Fečkan, M, Zhou, Y: Ulam's type stability of impulsive ordinary differential equations. J. Math. Anal. Appl. 395 258-264 (2012)

13. Wang, J, Fečkan, M, Zhou, Y: On the new concept of solutions and existence results for impulsive fractional evolution equations. Dyn. Partial Differ. Equ. 8, 345-361 (2011) 
14. Kilbas, AA, Srivastava, HM, Trujillo, JJ: Theory and Applications of Fractional Differential Equations. North-Holland Mathematics Studies, vol. 204. Elsevier, Amsterdam (2006)

15. Wang, J, Zhou, Y: A class of fractional evolution equations and optimal controls. Nonlinear Anal., Real World Appl. 12, 262-272 (2011)

16. Wang, J, Zhou, Y, Fečkan, M: Alternative results and robustness for fractional evolution equations with periodic boundary conditions. Electron. J. Qual. Theory Differ. Equ. 2011, 97 (2011)

10.1186/1687-2770-2014-35

Cite this article as: Yu and Wang: Periodic BVPs for fractional order impulsive evolution equations. Boundary Value Problems 2014, 2014:35

Submit your manuscript to a SpringerOpen ${ }^{\odot}$ journal and benefit from:

- Convenient online submission

- Rigorous peer review

Immediate publication on acceptance

- Open access: articles freely available online

- High visibility within the field

- Retaining the copyright to your article

Submit your next manuscript at $>$ springeropen.com 